\title{
Papel da psicologia no acompanhamento do paciente com diabetes
}

\author{
Sylvia M. Lima ${ }^{\text {* }}$
}

\section{Resumo}

O presente estudo foi desenvolvido a partir de revisão bibliográfica de temas relacionados ao diabetes mellitus, abordando a dificuldade de aceitação e a implicação da não adesão ao tratamento, que contribuem para pior controle metabólico e possível desenvolvimento de complicações, com aumento da morbidade e mortalidade e piora na qualidade de vida do paciente. Buscou-se esclarecer como a doença é recebida e percebida pela família e o seu importante papel de cuidadora, assim como a distribuição dos novos papéis sociais que, a partir do diagnóstico, são atribuídos aos seus membros. A abordagem desse estudo caracteriza-se, ainda, por ressaltar a importância de se cultivar uma boa relação médico-paciente, na qual o enfermo se sinta apoiado, confiante e se envolva com o seu tratamento. A necessidade de se estabelecer vínculos sólidos e seguros do paciente e de sua família com a equipe de saúde justifica-se por possibilitar uma melhor adesão ao tratamento. Doenças crônicas, como o diabetes mellitus, causam instabilidade emocional, gerando raiva, revolta, medo, tristeza e insegurança, suscitando mudanças e adaptações, com repercussões para o paciente e sua família. O trabalho desenvolvido pela psicologia médica consiste em facilitar uma boa relação médico-paciente. O atendimento psicológico deve ser prestado ao doente e à sua família, acompanhando-os desde o diagnóstico e, principalmente, durante o tratamento, oferecendo suporte na evolução da doença. A instabilidade emocional vivida pelo paciente com diabetes e o efeito da doença na família passa por fases de adaptação e aceitação. O acompanhamento psicológico, portanto, permitirá identificar e tratar a ansiedade, depressão, desajustes sociais, isolamento social e outros transtornos apresentados por alguns pacientes no curso do tratamento do diabetes mellitus.

Descritores: Diabetes mellitus; Família; Relações médicopaciente; Psicologia médica.

\section{Abstract \\ Psychology's role in patient monitoring with diabetes}

This study was a review of the literature including issues related to diabetes mellitus, addressing the difficulty of acceptance and non-adherence to treatment, which may contribute to a poor metabolic control and development of complications as a consequence with increased morbidity and risk of mortality and poor quality of life. We sought to clarify how the disease is received and perceived by the patient's family and its important role of a caregiver, as well as the new social roles that are allocated to its members since the diagnosis of diabetes. The
1. Ambulatório de Diabetes e Metabologia. Policlínica Piquet Carneiro. Universidade do Estado do Rio de Janeiro. Rio de Janeiro, RJ, Brasil.

*Endereço para correspondência: Policlínica Piquet Carneiro, UERJ Av. Marechal Rondon, 381

Rio de Janeiro, RJ, Brasil. CEP: 20950-003.

E-mail: sylviamlima@yahoo.com.br

Revista HUPE, Rio de Janeiro, 2015;14(4):76-80 doi: 10.12957/rhupe.2015.20062

Recebido em 28/11/2014. Aprovado em 07/01/2015.

approach of this study is also characterized by emphasizing the importance of cultivating a good physician-patient relationship where the patient feels supported, confident and get involved in the treatment. The need to establish strong links between the patient and his family and the healthcare staff is justified by better adherence to treatment. Chronic diseases such as diabetes mellitus, cause emotional instability, generating anger, fear, sadness and insecurity, prompting changes and adaptations with repercussions for the patient and his family. The role of the Medical Psychology is to facilitate a good physician-patient relationship. Psychological care should be provided to the patient and his family, following them since diagnosis and especially during treatment, supporting the progression of the disease. Emotional instability experienced by the patient with diabetes and the effect of the disease on the family goes through phases of adaptation and acceptance. The psychological treatment, therefore, allows to identify and treat anxiety, depression, social misfits, social isolation and other disorders presented by some patients in the course of diabetes mellitus treatment.

Keywords: Diabetes mellitus; Family; Physician-patient relationship; Psychology, medical.

\section{Resumen}

\section{Papel de la psicología en el seguimiento de pacien- tes con diabetes}

Este estudio fue desarrollado a partir de la revisión bibliográfica de temas relacionados con la diabetes mellitus, frente a la dificultad de aceptación, implicación y falta de adherencia al tratamiento, que contribuyen a empeorar el control metabólico y posible desarrollo de complicaciones, con aumento de 
la morbilidad, mortalidad y empeoramiento en la calidad de vida del paciente. Se trató de aclarar cómo la enfermedad es recibida y percibida por la familia, y su importante papel de cuidadora, así como la distribución de los nuevos roles sociales que, desde el diagnóstico, son asignados a sus miembros. El enfoque de este estudio se caracteriza por resaltar la importancia de cultivar una buena relación médico-paciente, en la que el paciente se sienta apoyado, confiado y se involucre con su tratamiento. La necesidad de establecer vínculos sólidos y seguros del paciente y su familia con el personal de salud se justifica, ya que permite una mejor adhesión al tratamiento. Las enfermedades crónicas, como la diabetes mellitus, causan inestabilidad emocional, generando rabia, indignación, miedo, tristeza e inseguridad, provocando cambios y adaptaciones, con repercusiones para el paciente y su familia. El trabajo

\section{Diabetes mellitus}

O diabetes mellitus (DM) é uma doença crônica, tendo como causa fatores genéticos (herdados) e ambientais. Os principais sintomas são: sede intensa, urinar com frequência, fome excessiva, rápida perda de peso, falta de energia, visão turva, cansaço extremo, físico e mental.É preciso que haja um bom controle da doença, para evitar complicações, como problemas de visão (retinopatia), problemas nos rins (nefropatia), problemas neurológicos (neuropatia) e cardiovasculares, sendo necessário seguir a prescrição médica, dieta, atividades físicas e manter um bom equilíbrio emocional. ${ }^{1}$

Doenças crônicas não transmissíveis representam um grupo de doenças com história natural prolongada, caracterizada por: múltiplos fatores complexos; interação de fatores etiológicos desconhecidos; longo período de latência; longo curso assintomático; manifestações clínicas, em geral de curso crônico, com períodos de remissão e exacerbação e evolução para incapacidades. ${ }^{2}$ Sua importância reside não somente na extensão dos danos físicos causados às pessoas acometidas por elas, mas também no impacto social e psicológico que provocam. $^{2}$

O modo como cada indivíduo enfrenta a doença está relacionado com a percepção que ele tem de saúde, a partir de suas experiências pessoais, suas crenças, valores e a forma como ele vivenciou essas experiências em seu âmbito familiar, ao longo de sua vida. ${ }^{3}$

É importante ressaltar a necessidade do paciente com diabetes ser acompanhando por uma equipe multiprofissional, onde cada profissional poderá contribuir com a sua experiência dando suporte ao paciente e à sua família.

A prevenção, bem como o manejo das compli- desarrollado por la psicología médica consiste en facilitar una buena relación médico-paciente. La atención psicológica debe proporcionarse al paciente y a su familia, acompañándolos desde el diagnóstico y, en especial durante el tratamiento, ofreciendo apoyo en la evolucion de la enfermedad. La inestabilidad emocional experimentada por el paciente con diabetes y el efecto de la enfermedad en la familia pasa por fases de adaptación y aceptación. El acompañamiento psicológico permitirá identificar y tratar la ansiedad, depresión, desajustes sociales, aislamiento social y otros trastornos que presentan algunos pacientes en el curso del tratamiento de la diabetes mellitus.

Palabras clave: Diabetes mellitus; Familia; Psicología Médica.

cações do DM são prioridade para a saúde pública mundial, nos diferentes níveis de atenção à saúde. ${ }^{4}$ $\mathrm{Na}$ atenção primária, através da educação em saúde, levando informações e orientação aos indivíduos em situação de risco; na atenção secundária, identificando os casos ainda não diagnosticados e possibilitando o seu tratamento; e, na atenção terciária, através do tratamento daqueles já acometidos pela doença, objetivando prevenir complicações e comorbidades. ${ }^{4}$

\section{Adesão ao tratamento}

De acordo com a Organização Mundial de Saúde (OMS), a adesão ao tratamento proposto implica na mudança de comportamento do indivíduo, no sentido de corresponder às recomendações de um profissional de saúde, isto é, tomar a medicação prescrita, seguir a dieta e mudar seu estilo de vida. ${ }^{5}$ A não adesão é causa importante de complicações, com aumento da morbidade e mortalidade, piora na qualidade de vida, excesso de procedimentos e utilização dos serviços de saúde com aumento dos custos médicos.

Segundo a OMS, cinco fatores influenciam na adesão: fatores socioeconômicos; fatores relacionados ao paciente (sexo, idade, estado civil, escolaridade e nível socioeconômico); fatores relacionados à doença (cronicidade, ausência de sintomas, percepção da seriedade do problema e experiência com a doença no contexto familiar); fatores relacionados ao tratamento (custos, efeitos indesejáveis, ineficácia de tratamentos, esquemas terapêuticos complexos e tempo de espera e de atendimento); e fatores relacionados aos sistemas e equipe de saúde (serviços de saúde, relação médico-paciente e o equilíbrio desta relação assimétrica). ${ }^{5}$

Estimular a adesão ao tratamento é de extrema im- 


\section{Artigo de revisão}

portância e a educação em saúde pode ser considerada uma das estratégias que possibilitam melhor adesão dos pacientes ao esquema de tratamento. Nesse sentido, a educação em saúde tem sido muito valorizada e é considerada parte integrante do tratamento das doenças crônicas, devendo-se levar em consideração os aspectos culturais, sociais, interpessoais e as reais necessidades psicológicas das pessoas com diabetes. ${ }^{6}$

A adesão ao tratamento não só beneficia ao paciente como também à sua família, aos sistemas de saúde e à economia dos países. A aceitação, o autocuidado e a adesão permitem que o paciente possa ter sua condição controlada, podendo desfrutar de uma melhor qualidade de vida e ser economicamente ativo e produtivo, reduzindo a incidência e a progressão das complicações da doença, bem como os custos para o sistema de saúde. Essa mudança de atitude diminui as complicações do diabetes e comorbidades, reduzindo o número de internações e intervenções cirúrgicas, permitindo a liberação da família do papel de cuidadora e evitando que o paciente interrompa suas atividades, com o seu afastamento do convívio social e familiar.

A educação em saúde deve levar em consideração a realidade e a vivência dos pacientes, permitindo maior participação e considerando o que eles já sabem e o que desejariam saber, tornando-os participativos na condução do seu tratamento. ${ }^{6}$

Para que esse processo educativo seja eficazé necessário conhecer suas crenças, sentimentos, pensamentos e comportamentos, bem como a forma que os mesmos interagem com o meio ambiente e com os outros indivíduos. Só assim será possível estabelecer estratégias de intervenção que possibilitem a adesão. ${ }^{6}$

\section{A doença na família e o papel do cuidador}

O surgimento da doença traz consigo vários significados simbólicos, a imagem corporal é atingida e isso pode provocar, além do sofrimento físico, sentimentos de desamparo, medo e culpa, fazendo com que o paciente regrida a um nível mais primitivo de funcionamento, gerando muita ansiedade.

Numa família em que o filho é portador de diabetes, torna-se necessário fazer uma readaptação, pois essa criança não corresponde à imagem que seus pais planejaram para ela, gerando grandes frustrações. ${ }^{1}$ Seus pais se sentem ansiosos, culpados, superprotegendo a criança, compensando assim a sobrecarga que experimentam com o tratamento. Vivem um luto intenso pela criança sadia, que é perdida quando se formaliza o diagnóstico. ${ }^{1}$
O diabetes pode provocar sentimentos de menosvalia, inferioridade, baixa autoestima, medo, revolta, raiva, ansiedade, regressão, negação da doença, desesperança, incapacidade de amar e se relacionar bem com as pessoas, ideias de suicídio e depressão. ${ }^{1}$

O diabetes será enfrentado diferentemente por cada indivíduo, pois dependerá da estrutura psíquica ou organização mental de cada um. A família também enfrenta a ansiedade da perda frente à enfermidade, que é dramática e exige cuidados, e por isso o diabetes deve ser tratado no grupo familiar, paciente e familiares apoiando-se mutuamente. ${ }^{1}$

Os familiares de pacientes diabéticos reagem com angústia e desespero perante a sensação de terem pouco controle sobre suas vidas e sobre a vida do paciente com diabetes. ${ }^{7}$ Enfrentam momentos de incerteza e descrença, intercalados por aceitação, perseverança, otimismo e esperança, que envolvem aspectos emocionais, afetivos, psicológicos, sociais, culturais e espirituais.?

Se o sistema familiar for flexível e aberto, com algumas modificações ele se adaptará à nova situação criada pela doença. Porém, se for rígido e fechado, a nova situação será percebida como uma ameaça ao seu equilíbrio, acarretando a rejeição do doente ou a sua utilização, sendo expressa através de formações reativas, superproteção e ansiedade.

A família, na medida em que serve como fonte de apoio emocional, pode ajudar o paciente a manejar a doença e alcançar as metas do seu tratamento?

As interferências causadas pelas reações emocionais emergem da necessidade de cuidados incessantes e repetitivos para o controle do DM e como expressão da percepção individual e emocional, causando maior dificuldade para o desempenho das ações contidas nos papéis de autocuidado e, consequentemente, para a adesão ao tratamento. ${ }^{8}$

A família é um fator preponderante para a aceitação da doença. Ao se sentir apoiado por sua família, que participa do seu tratamento e encara a situação com certa tranquilidade, o paciente com diabetes sente-se mais seguro e confiante em prosseguir em seu tratamento.

Daí a importância do suporte social, que proporciona ao indivíduo um sentimento de apoio, proteção e acolhimento, sendo capaz de reduzir o estresse e gerar bem-estar psicológico.

\section{A relação médico-paciente}

Michael Balint, psicanalista e bioquímico húngaro, formou-se em medicina em 1918, com uma brilhante carreira. Em seus seminários de pesquisa, realizados 
na Clínica Tavistock, em Londres, trabalhava como psiquiatra na supervisão de grupos clínicos, desenvolvendo uma prática médica de treinamento conhecida como Grupo Balint, em que eram estudadas as implicações psicológicas da clínica médica. Nessas reuniões, Balint supervisionava a atividade clínica de um grupo de médicos, no qual as experiências de todos eram discutidas em apresentações de casos clínicos, dando-se ênfase à relação médico-paciente.

Os médicos eram estimulados a examinar as próprias emoções e o sentido das reações e atitudes que se desenvolviam durante os processos de diagnósticos e dos tratamentos, pois, segundo Balint, desde o diagnóstico até a terapêutica e o prognóstico, todos os momentos dos atos médicos estão impregnados de sentimentos que podem ser úteis ou prejudiciais ao doente. Segundo Balint, a personalidade do médico é o primeiro "medicamento" que se administra aos pacientes. ${ }^{9}$

As discussões nos seminários revelaram que "a droga mais frequentemente utilizada na clínica geral era o próprio médico", isto é, não apenas importava o frasco de remédio, mas o modo como o médico o oferecia ao paciente, a forma como a substância era administrada e recebida. Num segundo momento, percebeu-se que não existe nenhum tipo de farmacologia a respeito de tão importante substância, pois em nenhum tipo de manual se encontrarão referências quanto a que dosagem o médico deve "prescrever a si mesmo", em que apresentação e posologia, qual suas doses de cura. ${ }^{9}$

Toda doença é também o "veículo" de um pedido de amor e atenção. ${ }^{9}$ A doença constitui, para o indivíduo, uma ameaça de dor, de invalidez e de morte. Desenvolve-se, por isso, um sentimento de insegurança e de necessidade de apoio que reedite a situação primitiva de relação da criança com a mãe. Todo paciente tem muito da criança medrosa que procura a mãe-médico em busca de apoio. ${ }^{9}$

É interessante ressaltar a importância do médico em demonstrar um interesse genuíno sobre o seu paciente, sobre os aspectos da sua vida, ter um entendimento global sobre o seu meio ambiente, sua vida familiar. As informações e relatos trazidos pelo paciente irão compor o contexto no qual ele se acha inserido. O médico deve ter uma escuta ativa para que possa realmente compreender o seu paciente e os aspectos que a doença acarreta em sua vida, deve ouvir de forma empática, acolhedora. Escutar para compreender, compreender para intervir e intervir de forma terapêutica, para que os resultados possam ser alcançados. Deve tratar o doente e não a doença.
Para o indivíduo, ser doente significa estar em situação de fraqueza, pois a doença representa quase sempre sofrimento, prejuízo corporal e limitação das possibilidades físicas, um golpe em sua integridade e um obstáculo às suas atividades diárias. Significa ainda estar em situação de dependência física e moral, frente ao seu grupo social. ${ }^{10}$

A doença representa um ataque à autoimagem ideal e uma prova de falibilidade. O doente perde bruscamente sua ilusão de onipotência, de invulnerabilidade. ${ }^{10}$

O médico tem um papel tranquilizador e reorganizador no atendimento ao seu paciente e pode, através de suas atitudes, aliviar o doente de suas tensões, permitindo que ele se reorganize. Porém, se for indiferente, contribuirá para aumentar a angústia do paciente fazendo com que ele se desorganize psiquicamente ainda mais. ${ }^{10}$

O médico deve estabelecer uma relação de empatia, desenvolvendo uma boa e eficiente comunicação, adaptando sua linguagem à do paciente. Orientá-lo sobre as opções para o seu tratamento, avaliando os riscos e benefícios, possibilitando a oferta de conhecimento sobre o paciente e a sua doença, simplificando a terapêutica.

Na relação médico-paciente, embora seja uma relação assimétrica, deve-se evitar a verticalização, na qual o profissional detém o conhecimento e o poder de decisão sobre a conduta a seguir. A comunicação deve ser clara e simples, para que o paciente sinta-se confiante e participe ativamente do seu tratamento, siga a prescrição e fique atento aos cuidados recomendados pelo seu médico, estabelecendo-se um vínculo em que atitudes positivas influenciem diretamente em sua saúde, implicando numa melhor adesão, no autocuidado e qualidade de vida.

\section{O papel da psicologia médica no tratamento do paciente com diabetes}

Receber o diagnóstico de DM acarreta muitas vezes em um choque emocional para a pessoa, que não está preparada para conviver com as limitações decorrentes da condição crônica, o que acaba interferindo em sua vida familiar e afetando suas relações. ${ }^{6}$

O indivíduo com doença crônica experimenta, de forma intensa, a ambivalência, isto é, precisa decidir entre aquilo que deseja fazer e o que é preciso ser feito. Nesse conflito existem ganhos e perdas, sentimentos ambíguos, gerando muita instabilidade emocional. Daí a necessidade de acompanhamento psicológico para que ele possa elaborar esses aspectos emocionais da doença, diminuindo assim seu sofrimento psíquico, 
possibilitando a aceitação, motivando-o no autocuidado e na adesão ao tratamento.

Mudança de hábitos exige motivação, planejamento e disciplina, uma rotina que às vezes fica difícil de ser cumprida e incorporada à vida diária pelo portador de diabetes. Sentimentos e crenças interferem na motivação, predispondo o paciente a transgredir, a burlar a dieta, atendendo ao seu "desejo", que ele confunde com fome e necessidade. O uso contínuo de medicamentos, consultas, exames, dieta e exercício físico são parte dessa mudança e, com o tempo, o paciente se queixa de estar cansado dessa rotina desgastante.

Cada indivíduo vai se apropriar de modo muito peculiar de sua doença. A percepção que tem de si mesmo, das dificuldades e das limitações com que se depara no curso da doença e de sua forma de enfrentamento é que permitirão que ele dimensione a situação e se capacite para a resolução de problemas.

O papel da psicologia médica consiste em intermediar a relação médico-paciente, facilitando esse diálogo. E, por ter uma escuta diferenciada, o psicólogo é capaz de intervir, de estreitar o vínculo entre o médico e seu paciente, criando condições para que o paciente se sinta acolhido e mais motivado em seu tratamento, livre de cobranças ou críticas, estabelecendo uma relação de parceria, participando, contribuindo e tendo mais controle sobre a sua doença, estimulando a presença de familiares do paciente na consulta, que poderão contribuir com informações, participando das discussões sobre o tratamento.

Trabalhar a aceitação da doença e a motivação do paciente para seguir a terapêutica, autocuidado, prática de atividade física, dieta e equilíbrio emocional fazem parte do trabalho da psicologia, através da psicoeducação e orientação, oferecendo acolhimento e suporte.

\section{Conclusão}

Em vista do que foi apresentado neste trabalho, destaca-se a prevenção e os cuidados que todos os indivíduos devem ter com a sua saúde antes que a do- ença se instale, tendo sido ressaltada a importância do tratamento ser feito por uma equipe multiprofissional.

Estimular a adesão, através da aceitação da doença, do trabalho de educação em saúde, da participação ativa da família e uma boa relação médico-paciente, contribuirá para uma melhor qualidade de vida, afastando o risco de complicações, excesso de procedimentos e internações e elevados custos médicos. O papel da psicologia consiste em trabalhar a aceitação e motivação do paciente, esclarecendo suas dúvidas, reestruturando suas crenças, sendo continente para seus medos, frustrações, expectativas e fantasias. Incentivando-o a ampliar sua rede social, oferecendo suporte e acolhimento, transmitindo-lhe apoio, segurança e confiança.

\section{Referências}

1. Marcelino DB, Carvalho MDB. Reflections on the diabetes type 1 and its relantion to the emotional aspect. Psicologia: Reflexão e Crítica. 2005;18(1):72-7.

2. Reiners AA, Azevedo RC, Vieira MA, et al. [Bibliographic production about adherence/non-adherence to therapy]. Cien Saude Colet. 2008 Dec;13 Suppl 2:2299-306.

3. Xavier ATF, Bittar DB, Ataíde MBC. Beliefs in diabetes selfcare- implications for practice. Texto \& Contexto-Enfermagem. 2009;18(1):124-30.

4. Zanetti ML, Otero LM, Freitas MCF, et al. Atendimento ao paciente diabético utilizando o protocolo Stage Diabetes Management: relato de experiência. RBPS. 2006;19(4):253-60.

5. World Health Organization. Adherence to long-term therapies: evidence for action. 2003 [12/10/2014]. Available from: http:// www.who.int/chp/knowledge/publications/adherence_full_report.pdf].

6. Peres DS, Santos MA, Zanetti ML, et al. Difficulties of diabetic patients in the illness control: feelings and behaviors. Rev Lat Am Enfermagem. 2007 Nov-Dec;15(6):1105-12.

7. Santos ECB, Zanetti ML, Otero LM, et al. Care according to diabetes patients and their main caregivers. Rev Lat Am Enfermagem. 2005;13(3):397-406.

8. Filho CVS, Rodrigues WHC, Santos RB. Self-Care rolesSubsidy to the Nursing before the emotional reactions of the diabetes mellitus carriers. Esc Anna Nery. 2008;12(1):125-9.

9. Balint M. O médico, seu paciente e a doença. Rio de Janeiro: Atheneu; 1975.

10. Jeammet P, Reynaud M, Consoli S. Manual de Psicologia Médica. São Paulo: Editora Masson do Brasil; 1989. 